Research Article

\title{
Nondestructive Detection of Moisture Content in Walnut Kernel by Near-Infrared Diffuse Reflectance Spectroscopy
}

\author{
Dan Peng $\mathbb{D}^{\mathrm{D}}$, Yali Liu, Jiasheng Yang, Yanlan Bi $\mathbb{D}$, and Jingnan Chen \\ College of Food Science and Technology, Henan University of Technology, Lianhua Road 100, Zhengzhou 450001, \\ Henan Province, China \\ Correspondence should be addressed to Dan Peng; pengdanhaut@126.com and Yanlan Bi; yanlanhaut@163.com
}

Received 4 March 2021; Accepted 9 June 2021; Published 17 June 2021

Academic Editor: Thomas Walther

Copyright (c) 2021 Dan Peng et al. This is an open access article distributed under the Creative Commons Attribution License, which permits unrestricted use, distribution, and reproduction in any medium, provided the original work is properly cited.

The rapid and accurate detection of the moisture content is of great significance to the quality evaluation and oil extraction process of walnut kernel. Near-infrared (NIR) spectroscopy is an ideal method for measuring the moisture content in walnut kernel. In this study, a regression model for moisture content in walnut kernel was developed based on NIR diffuse reflectance spectroscopy using chemometric methods. The different spectral pretreatment methods were adopted to preprocess the original spectral data. The whole spectra band was divided into 5 subbands, 10 subbands, 15 subbands, and 20 subbands to screen specific wavelengths relevant to the walnut kernel moisture content. PLS (partial least square regression), MLR (multivariate linear regression), PCR (principle component regression), and SVR (support vector regression) were used to establish the relationship model between the spectral data and measurement values of the moisture content. In comparison, the optimized modeling conditions were determined as follows: detection wavelength $1349-1490 \mathrm{~nm}, \mathrm{SNV}-\mathrm{FD}$ (standard normal variate transformation and first derivative) preprocessing method, and PLS algorithm. Under these conditions, the square correlation coefficient $\left(R^{2}\right)$ and root mean square error of prediction (RMSEP) of the prediction model were 0.9865 and 0.0017 , respectively. The results of this study provided a feasible method for the rapid detection of moisture content in walnut kernel. To improve the performance and applicability of the model, it is necessary to continuously expand the size of the sample set.

\section{Introduction}

The walnut is one of the most important special oils and woody oil crops, which has both ecological and economic value. The walnut kernel is not only rich in nutritional value (e.g., oil, proteins, carbohydrates, and minerals) but also contains some minor components (e.g., phenols, tocopherols, and phytosterols) with high antioxidant capacity $[1,2]$. China is a major consumer, producer, and exporter of walnut, with the walnut production of over 1.5 million tons in 2018 (FAO Statistics). Restricted by technologies such as shelling, drying, and peeling, the harvested walnuts cannot be processed as quickly as possible [3-5]. Consequently, the moisture content in walnut kernel becomes an important reference index for evaluating walnut kernel quality, choosing storage conditions and processing methods [6-8]. On the one hand, proper moisture can increase the oil extraction rate. The moisture content of walnut kernel should be less than 5.0\% according to LS/T 3121-2019 Walnut for Oil. On the other hand, to a certain extent, the level of moisture content determines the economic benefits for growers and the walnut business. Walnut kernels with low moisture content will be lighter and their prices will decrease, while high moisture content will accelerate the rate of oil oxidation and mildew, resulting in the deterioration of walnut quality [9-11]. Therefore, it is crucial to accurately detect the moisture content in walnut kernel.

The classical detection method for the moisture content is the drying method. The moisture in the walnut kernel can be divided into free water and equilibrium water according to whether it can be removed by the drying method. The moisture that can be removed by the drying method is free water, and the moisture studies in this study are free water. Due to its drawbacks of time-consumption, destruction, and 
complication, it is important to find a rapid, nondestructive, and "online" method, which could provide significant information. Near-infrared diffuse reflectance spectroscopy is a powerful tool to evaluate the quality parameters, which has been widely used in food, agriculture, chemical industry, biomedicine, and other fields [12-14]. With the recent development of spectroscopy technology, more and more NIR spectroscopy technologies have been used as national standards. The application of NIR spectroscopy in crop quality analysis has become increasingly popular [15-17]. A number of studies have shown that near-infrared spectroscopy technologies can be used to predict the moisture content of grains and oil seeds [18-20]. For example, NIRS technique has been successfully used for the rapid analysis of moisture content in wheat flour samples [21-24]. López A et al. used nearinfrared spectroscopy technologies to detect the moisture content in potato products $[25,26]$. NIR diffuse reflectance spectroscopy also allowed rapid and online analysis of the moisture content in cocoa beans samples [27, 28]. Moreover, Lakshmanan has suggested that NIRS could be applied to monitor moisture and oil moisture content at the reception in the mill about the quality and composition of the copra, which was useful in the copra oil extraction process [29]. When chemometric methods were used together with NIR, a quantitative model of chestnut moisture content could be easily established, with the root mean square error of cross-validation (RMSECV) of 0.05 and the coefficient of determination $\left(R^{2}\right)$ of 0.9 [30]. Zhang and Dai used NIR spectroscopy to establish a quantitative model of moisture content in corn seed and concluded that the model performance of a single variety of corn seed was greater than that of multiple varieties [31, 32]. However, there are fewer studies on detecting the moisture content in walnut kernel using NIR spectroscopy. Here, the main purpose of this study attempts to build a rapid quantitative method for moisture content measurement in walnut kernel using NIR spectroscopy, which was expected to provide technical support for the rapid detection and control of walnut kernel quality.

\section{Experiment}

2.1. Preparation of Walnut Kernel Samples. The walnut kernel samples used in the experiment were collected from Aksu, Xinjiang, China. The walnut variety was Wen 185. In order to make the walnut kernel samples more representative, the method of moisture absorption in a closed container was adopted. According to Lu's experimental method [27], the walnut kernels were crushed by a high-speed universal pulverizer and passed through a 10 -mesh fine sieve after removing the shell. The filtered samples were used in the experiment. A certain amount of walnut kernels were placed in a closed container with water at the bottom, and then, the container was stored in a constant temperature incubator at $20^{\circ} \mathrm{C}$ to make the water absorbed evenly. At last, a total of 136 walnut kernel samples with different moisture contents were prepared in this way.
2.2. Chemical Analysis. According to the Chinese national standard GB/T 14489.1-2008, the moisture content in walnut kernel was measured by the drying method. The moisture content of each sample was measured immediately after the spectral data were collected. Each sample was measured twice, and the average value was taken as the reference value as given in Table 1 . The coverage range of the moisture content was $1.20-9.92 \%$, with the average value of $5.55 \%$ and the standard deviation of 0.27 . The error margin of the drying method used for the sample was less than $8 \%$. The 136 walnut kernel samples were randomly divided into the calibration set and prediction set according to the ratio of $3: 1$, of which 102 samples were contained in the calibration set and the remaining samples were contained in the prediction set.

2.3. NIR Spectra Acquisition. NIR spectra of walnut kernel samples were recorded using the FOSS NIRS DS2500 spectrometer (FOSS, Denmark) system equipped with a tungsten halogen lamp. The detector was silicon $(780-1100 \mathrm{~nm})$ and lead sulfide $(1100-2500 \mathrm{~nm})$. NIR spectrum was recorded in the wavelength range $780-2500 \mathrm{~nm}$ (interval $2 \mathrm{~nm}$, and data points 860 per a sample). The operating conditions were listed as follows: the operating temperature $35^{\circ} \mathrm{C}$ and the number of scans 32 . The depth of walnut kernels was $20 \mathrm{~mm}$ thickness. All data were obtained in triplicate, and the mean value was used in subsequent calculations.

2.4. Statistical Analysis. The spectral data were preprocessed by standard normal variate transformation (SNV), multiplicative scatter correction (MSC), first derivative (FD), orthogonal signal correction (OSC), detrend, and normalization (normalize), which were for reducing the influences of background noise, baseline drift, and spectral scattering [33]. In model construction for the moisture content in walnut kernel, multiple linear regression (MLR), principal component regression (PCR), partial least squares regression (PLS), and support vector regression (SVR) have been applied to develop the corresponding models between spectral data and measurement values [34]. The performances of multivariate models were evaluated by the coefficients of determination $\left(R^{2}\right)$, the root mean square error of prediction (RMSEP), the root mean square error of calibration (RMSEC), and residual predictive deviation (RPD). All modeling computations were implemented using the Unscrambler X10.4 and Matlab v2007a.

\section{Results and Discussion}

3.1. Characteristics Analysis of Samples Spectral Data. The raw NIR spectra $(780-2500 \mathrm{~nm})$ of walnut kernel samples are plotted in Figure 1. It can be seen from Figure 2 that the basic variation trends of spectral curves are similar, and there are no obvious abnormal variation points found. All spectral curves have the spectral energy absorption at the wavelengths of $1210 \mathrm{~nm}, 1450 \mathrm{~nm}, 1725 \mathrm{~nm}, 1890 \mathrm{~nm}$, $2000 \mathrm{~nm}, 2328 \mathrm{~nm}$, and $2350 \mathrm{~nm}$, and the differences of these 
TABLE 1: The moisture content of walnut kernel samples.

\begin{tabular}{lcccc}
\hline Subsets & Sample number & Moisture content (\%) & Mean (\%) & Standard deviation $^{\mathrm{a}}$ \\
\hline Calibration set & 102 & $1.20-9.92$ & 5.57 & 0.26 \\
Prediction set & 34 & $1.43-9.43$ & 5.52 & 0.27 \\
Total samples & 136 & $1.20-9.92$ & 5.55 & 0.27 \\
\hline
\end{tabular}

${ }^{a}$ The standard deviation refers to the spread of the individual samples.

near-infrared absorption peaks are mainly due to the differences in the internal component contents of these samples. Among these, the spectra present the dominant absorption peaks at $1450 \mathrm{~nm}$ and $1940 \mathrm{~nm}$, which are assigned to the first overtone and combination overtone of $\mathrm{O}-\mathrm{H}$ stretching vibration. In fact, they are indeed closely related to the moisture contents in walnut kernel [35]. Although the near-infrared spectra of different walnut kernel samples are obviously different, there still exist spectral overlaps and background interference. Modeling directly from the original spectrum must affect the model's robustness and accuracy. Therefore, the modeling conditions need to be optimized, and the spectrum also needs to be preprocessed.

\subsection{Optimization of Modeling Conditions}

3.2.1. Screening of Pretreatment Methods. The original spectral signal collected by the instrument not only contains information related to the chemical composition of the sample but also contains irrelevant interference information such as baseline drift, sample physical properties, background, and noise. These interferences directly affect the accuracy of the final analysis result. To improve the prediction precision of the moisture content model, normalize, $\mathrm{FD}, \mathrm{SNV}$, detrend, MSC, and OSC were used to preprocess the original spectral data. The results are shown in Figure 2. It is clear that there are certain differences between the preprocessed spectra with different algorithms. Among them, the profiles of spectra after normalize, MSC, and SNV are not significantly different from that of original spectra. However, the degree of the spectrum dispersion is significantly reduced, which highlighted the useful information in some bands. On the contrary, FD, detrend, and OSC cause significant changes in spectral morphology. The FD algorithm can eliminate the interference caused by the background and baseline drift, but at the same time, it also can cause noise amplification. The detrend algorithm can avoid the influence of trends, while the OSC algorithm can filter out part of the noise in the original spectrum and retain the main information. Although different pretreatment methods have the ability to eliminate information irrelevant to the analyte component, different measurement systems suffer from different interference factors. Therefore, it is necessary to choose the appropriate pretreatment method in the modeling phase. The PLS-based results are given in Table 2.

It can be seen from Table 2 that the pretreatment methods have a certain impact on the performance of the calibration model. Except for the OSC method, most

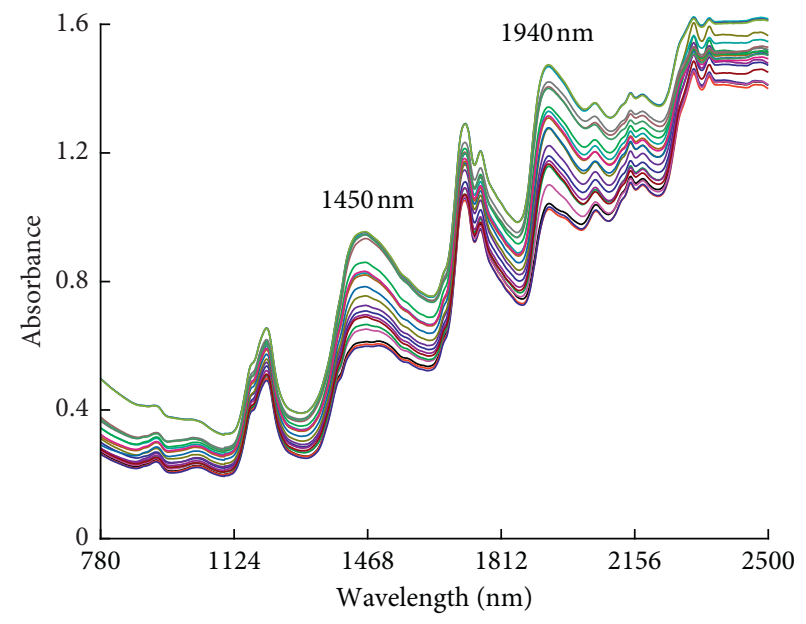

FIGURE 1: Near-infrared diffuse reflectance spectra of walnut kernel samples.

methods can improve the prediction precision of the built models. By comparing models with the single pretreatment method, the model with the normalize algorithm has the best prediction precision. The value of RMSEP is 0.0024 , which is improved by $33.3 \%$ compared with that of the model only with original spectra. This is due to that the normalize algorithm can effectively reduce the interference of invalid variable information and highlight the valid information related to moisture in the band. The OSC algorithm can only filter out their projections in the direction orthogonal to analyte, but the noise in the spectral data is not absolutely orthogonal to analyte. So, the residual part of the noise may cause overfitting and would further affect the stability of the model. Additionally, both the MSC algorithm and the SNV algorithm can reduce baseline drift and spectral scattering, which have the similar improvement on prediction accuracy. The FD algorithm also can eliminate the baseline drift and background interference of the spectra, but it amplifies the influence of some noise information, leading to the loss of part of the effective information and the deterioration of prediction accuracy. When combining two of the five algorithms as a new pretreatment algorithm, SNV-FD was deemed to be the best pretreatment method. The $R^{2}$ in calibration and prediction are both greater than 0.98 . The RPD value is 7.40 , which is the maximum value of several methods. Moreover, the value of RMSEP is 0.0020 , which is improved by $44.4 \%$ compared with that of the model only with original spectra and by $16.7 \%$ compared with that of the model with the best single pretreatment algorithm. Here, the optimized prediction model of walnut kernel moisture content was established using the SNV algorithm and the first derivative algorithm. Through analysis, it was also found 


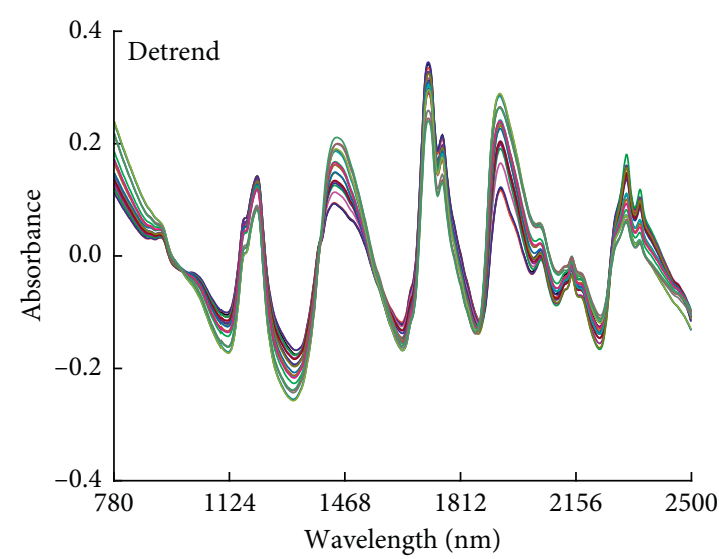

(a)

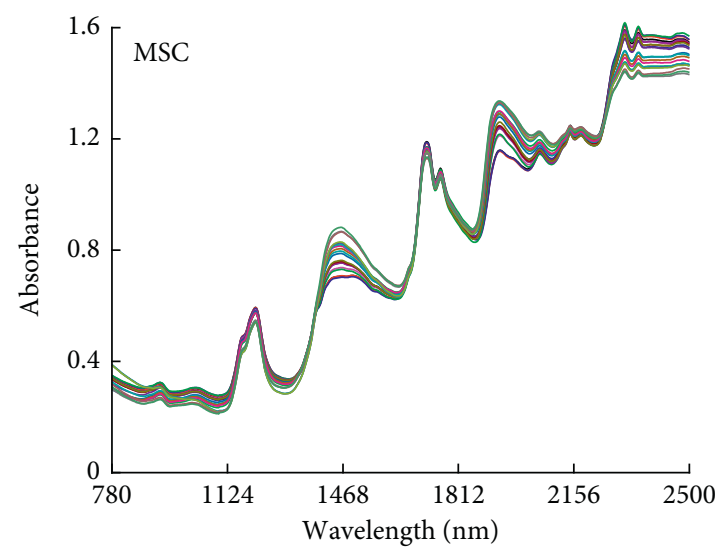

(c)

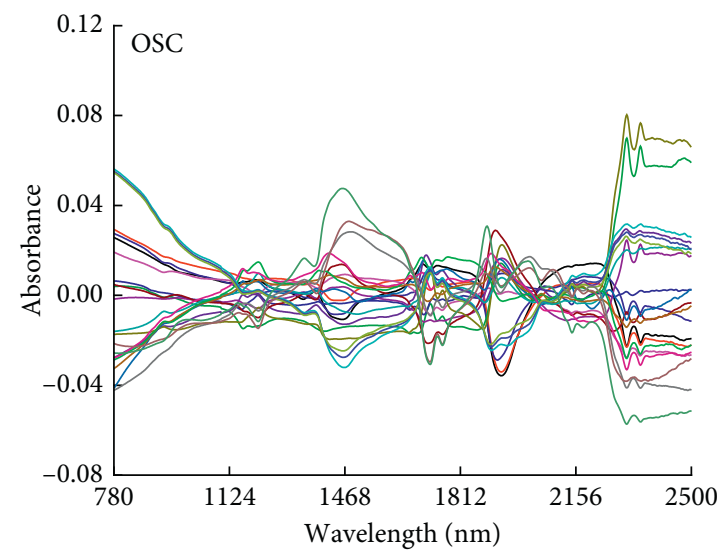

(e)

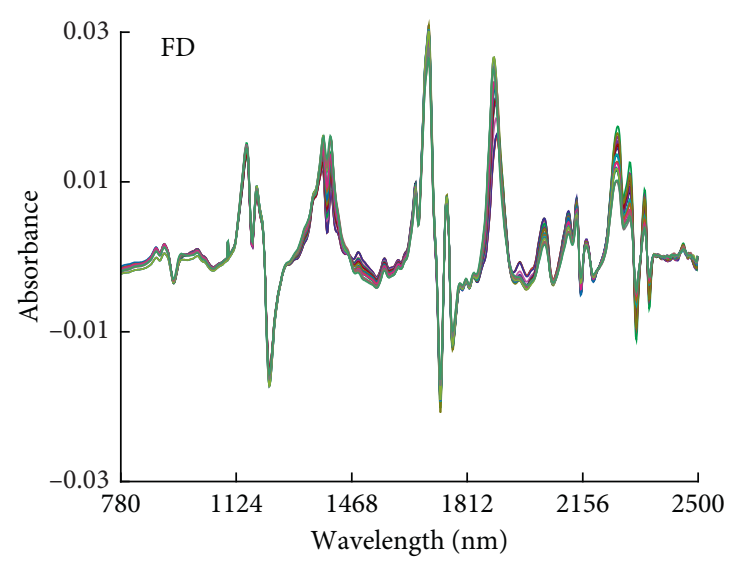

(b)

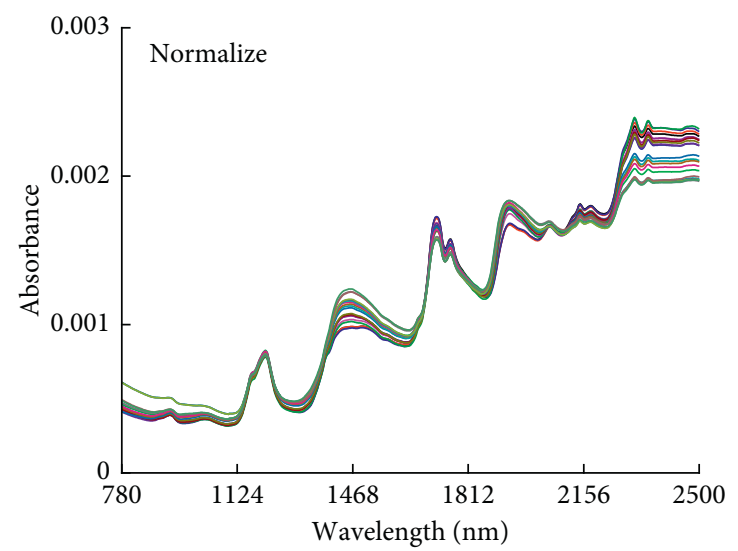

(d)

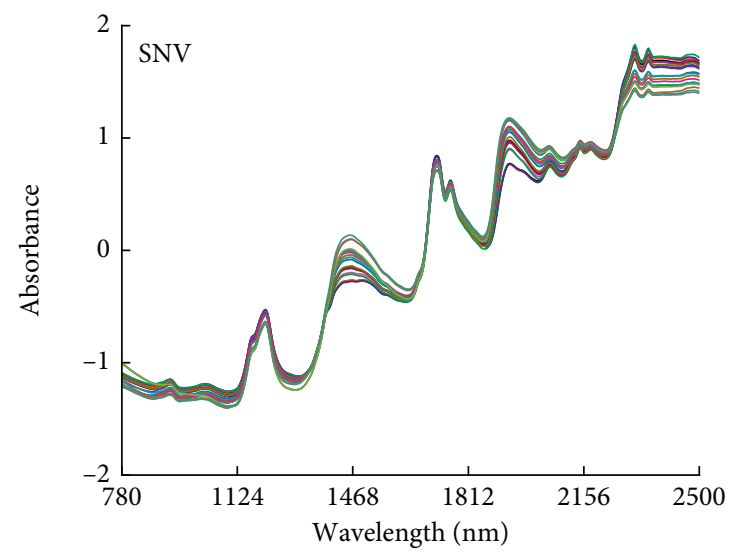

(f)

Figure 2: The near-infrared diffuse reflectance spectra of walnut kernel with different pretreatment methods.

that the combination of more than two pretreatment algorithms was not necessary for further performance improvement.

3.2.2. Selection of Characteristic Band. For the NIR spectrum of the moisture content in walnut kernel, there are a large number of wavelengths irrelevant to the moisture content, which could cause a great interference to moisture content measurement. Selecting specific wavelengths relevant to the analyte can significantly reduce the number of input variables. This will mainly bring two benefits. On the one hand, band selection can avoid instrument hardware interference because the presence of interference signals such as the noise makes the signal-to-noise ratio of some bands low and the quality of the collected spectrum poor. On the other hand, a small number of variables are beneficial to effectively extract information and eliminate noninformation [36]. Here, the whole spectra band is divided into 5 subbands, 10 subbands, 15 subbands, and 20 subbands, 
TABLE 2: Results of the calibration model for moisture content in walnut kernel with different pretreatment methods.

\begin{tabular}{lccccc}
\hline Pretreatment & $\mathrm{nPC}$ & $R_{\mathrm{c}}^{2}$ & RMSEC & $R_{\mathrm{p}}^{2}$ & RMSEP \\
\hline Original spectrum & 3 & 0.9437 & 0.0035 & 0.9377 & 0.0036 \\
Normalize & 3 & 0.9759 & 0.0023 & 0.9724 & 0.0024 \\
FD & 3 & 0.9719 & 0.0025 & 0.9701 & 0.0025 \\
MSC & 4 & 0.9765 & 0.0023 & 0.9723 & 0.0024 \\
SNV & 2 & 0.9740 & 0.0024 & 0.9705 & 0.0025 \\
Detrend & 3 & 0.9730 & 0.0025 & 0.9713 & 0.0025 \\
OSC & 5 & 0.1724 & 0.0132 & 0.1665 & 0.0136 \\
Normalize + MSC & 2 & 0.9739 & 0.0024 & 0.9705 & 0.0025 \\
Normalize + detrend & 3 & 0.9792 & 0.0022 & 0.9772 & 0.0022 \\
Normalize + SNV & 2 & 0.9740 & 0.0024 & 0.9705 & 0.0025 \\
Normalize + FD & 2 & 0.9732 & 0.0024 & 0.9710 & 5.92 \\
MSC + detrend & 3 & 0.9730 & 0.0025 & 0.9713 & 0.0025 \\
MSC + SNV & 2 & 0.9740 & 0.0024 & 0.9705 & 0.0025 \\
MSC + FD & 3 & 0.9720 & 0.0025 & 0.9701 & 0.0025 \\
Detrend + SNV & 3 & 0.9807 & 0.0021 & 0.9787 & 0.0025 \\
Detrend + FD & 3 & 0.9701 & 0.0026 & 0.0021 \\
SNV + FD & 3 & 0.9858 & 0.0018 & 5.92 \\
\hline
\end{tabular}

respectively. Coupled with the SNV + FD algorithm, the PLS models of moisture content are established as shown in Figure 3. It can be seen that the model divided into ten subbands had the best prediction precision, and the coverage range of RMSECV was $0.0023-0.0035$. This is probably due to that the wider spectrum area could introduce more interference information, while the narrower spectrum area may lose the analyte information and reduce the prediction accuracy of the model. Among these ten subbands, the model based on subband $5(1349-1490 \mathrm{~nm})$ can get the best prediction precision, mainly due to the strong absorption peak of -OH (near $1450 \mathrm{~nm}$ ) in water. The values of $R^{2}$ and the RMSECV are 0.9845 and 0.0023 , respectively, meaning that the prediction precision is improved by $32 \%$ compared to that of the model established with the whole band. Therefore, subband 5 is chosen as the modeling band for moisture content measurement.

3.2.3. Comparison of Modeling Methods. General modeling methods include linear calibration methods (PLS, MLR, PCR, and so on) and nonlinear calibration methods (SVR, artificial neural networks, and so on) in near-infrared spectroscopy analysis [34]. In this study, four representative methods including MLR, PLS, PCR, and SVR were applied to develop the appropriate regression model for walnut kernel moisture content measurement. The corresponding performances are shown in Figure 4.

It can be seen from Figure 4 that there are significant differences in the prediction performance of the four modeling methods. The prediction results of linear modeling (PLS, PCR, and MLR) are significantly better than that of nonlinear modeling (SVR). This was mainly because that there is a good linear relationship between the moisture content and spectral information in nature. However, the SVR algorithm is a nonlinear modeling method and cannot effectively process the information related to the moisture content. Among these linear modeling methods, the PLS model has the best performance with $R^{2}$ equal to 0.9865 and RMSEP equal to 0.0017 , showing that the prediction precision can be improved by $60.5 \%$ compared to that of the SVR model. The reason may be that the PLS algorithm introduces the concentration information of the measured component into the decomposition process of the spectrum matrix, and the scores of the spectral matrix and the concentration matrix are exchanged before each principal component is calculated. Consequently, the principal component of the spectral matrix is associated with the concentration of the measured component. However, PCR can eliminate useless noise information, but the influence of the measured component concentration is not considered during the decomposition of the spectral matrix [37]. In the MLR method, good results rely on the requirements that the input variables should be independent of each other, and the treatment effect of multicollinearity between variables should be poor. In fact, these requirements are impossible in complex spectra. Therefore, the PLS method is chosen for moisture content model construction.

3.3. External Validation of the Quantitative NIRS Models. In order to validate the stability and accuracy of the model, the PLS, MLR, PCR, and SVR calibration models were built to predict the moisture content of 30 unknown samples as shown in Figure 5. The data for Figure 5 have been performed double-blind.

It is shown in Figure 5 that the performance of the PLS model is excellent. The prediction precision can be improved by $56.4 \%$ than that of the SVR model. This is mainly because the PLS algorithm considers the concentration matrix and spectrum matrix simultaneously, and the multilinearities between spectra are reduced. Moreover, the paired $t$-test was applied to judge whether there was a significant difference between the near-infrared spectroscopy method and the national standard method. The $t$-test result showed that the $P$ value was 0.704 , indicating that the two methods had no significant difference (with criterion $\alpha=0.05$ and 


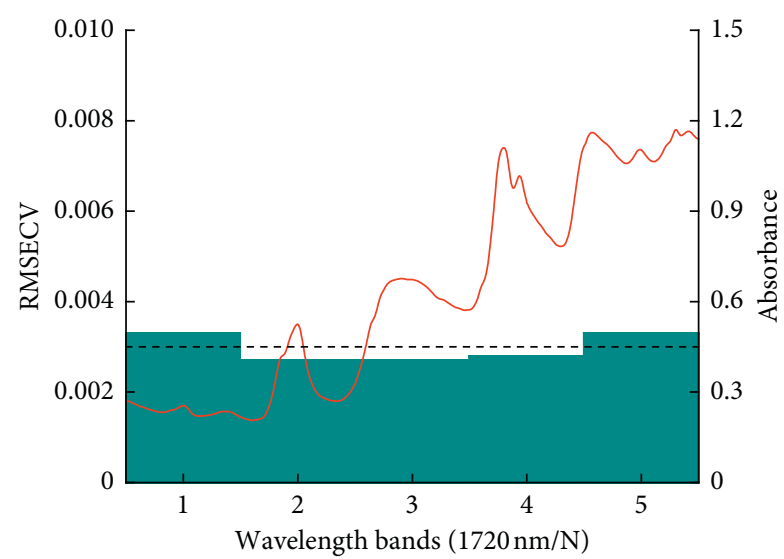

RMSECV

--- Average value

(a)

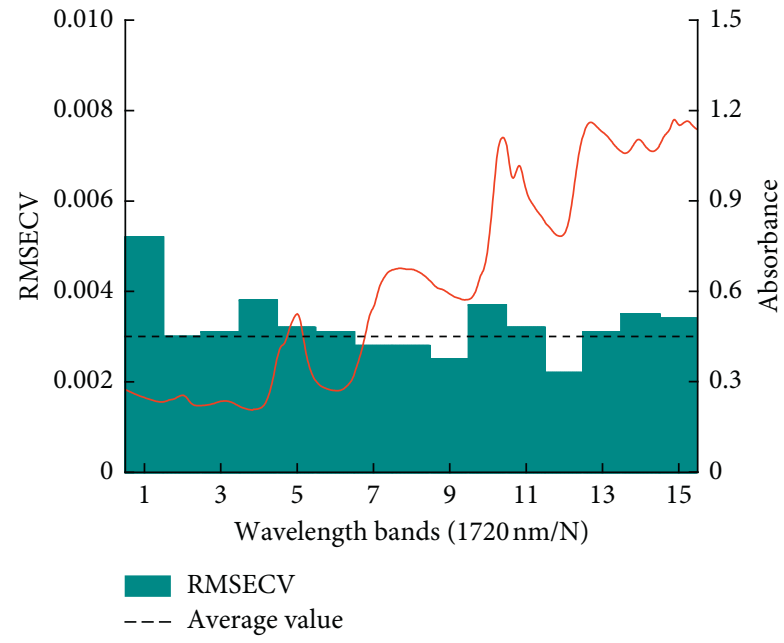

(c)

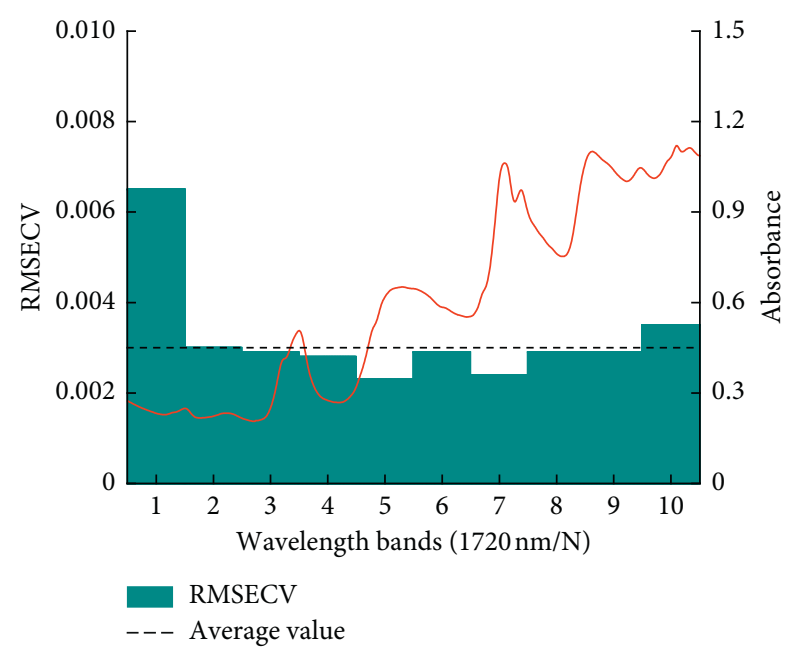

(b)

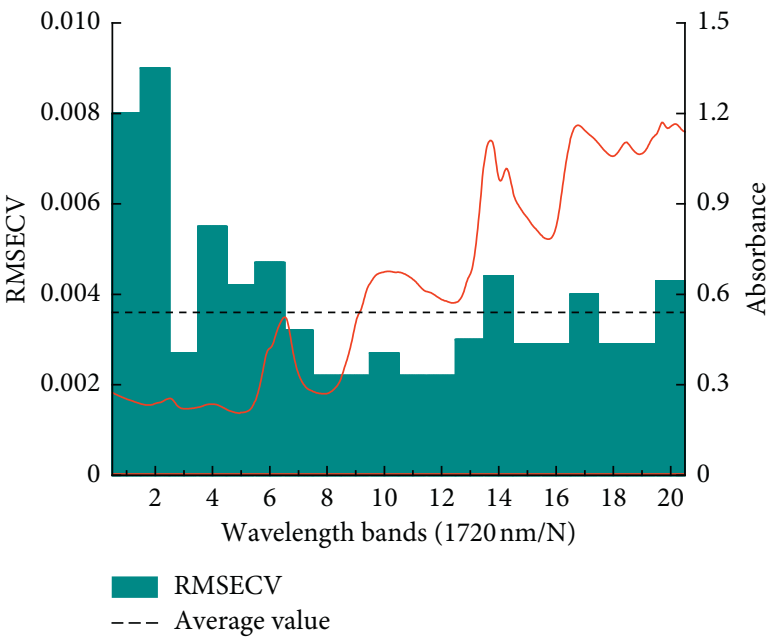

(d)

FIGURE 3: Prediction results of walnut kernel moisture content in different spectral bands.

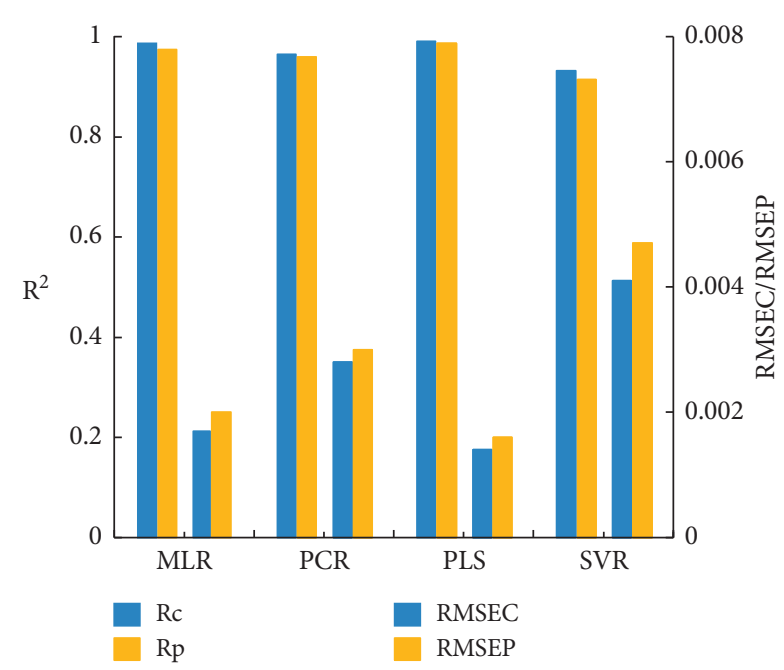

FIGURE 4: Prediction results of moisture content in walnut kernel using different modeling methods. assumption that there was a significant difference between the true value and the predicted value). Through comparison, the results obtained in present work are quite well comparable with the accuracy of online equations for the moisture content in coco-peat reported by Lu and coworkers $\left(R^{2}=0.99\right.$; $\left.\mathrm{RMSEP}=0.014\right)$ [27]. The current research also shows a quite lower RMSEP value (0.0017) than that recorded by Salguero-Chaparro and coworkers (0.016) in the prediction of the moisture content in olive fruits [38]. In addition, the $R^{2}$ and RMSEP values of the prediction model in this work are greater than those observed by $\mathrm{Hu}$ and coworkers $\left(R^{2}=0.90 ; \mathrm{RMSEP}=0.05\right)$ for the moisture content in the chestnut moisture content [30]. Therefore, it can be concluded that it is feasible to use near-infrared diffuse reflectance spectroscopy to measure the moisture content in walnut kernel. However, walnut kernels show different moisture contents due to different varieties, origins, and cultivation managements, as well as changes along with storage conditions and time after harvest, which limit the 


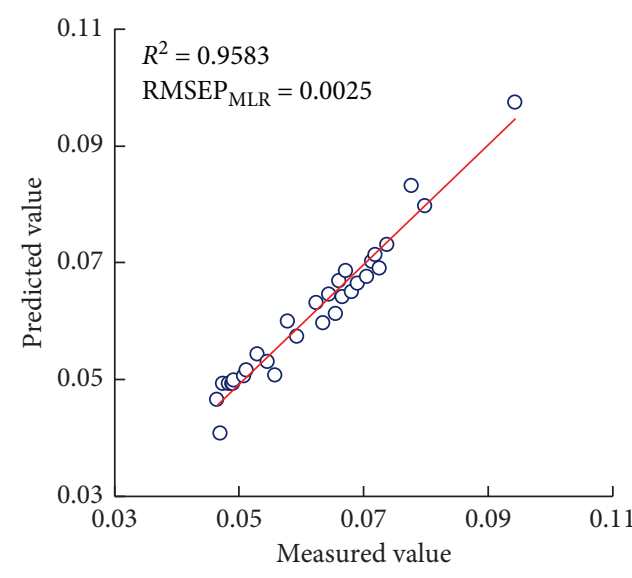

(a)

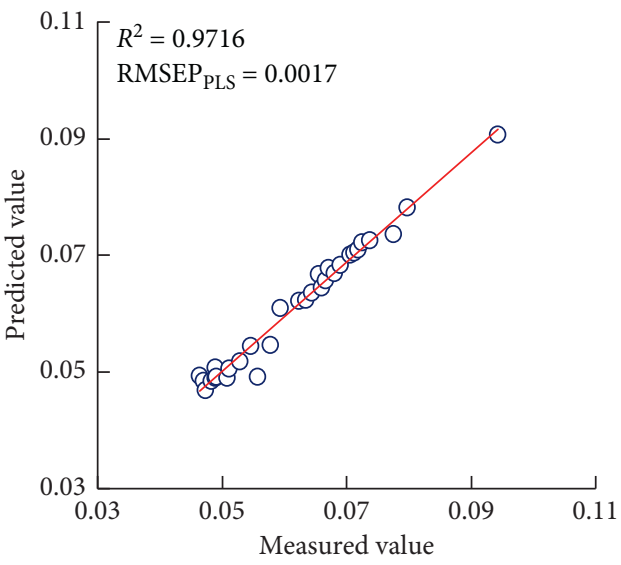

(c)

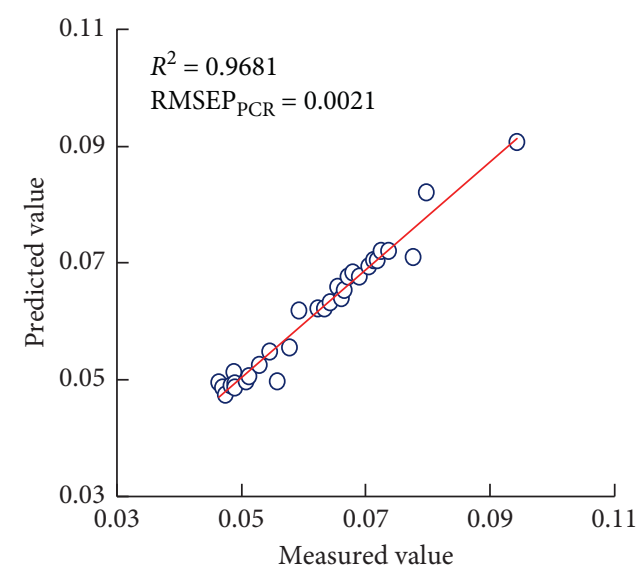

(b)

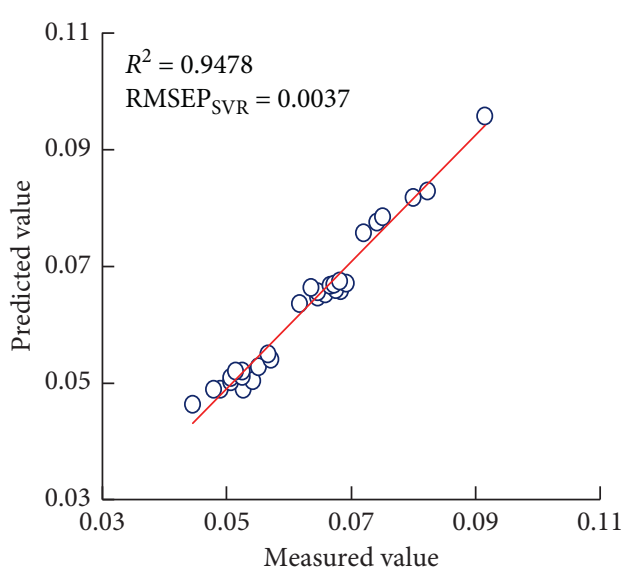

(d)

FIGURE 5: Prediction results of moisture content in walnut kernel.

applicability of this model. Thus, to improve the adaptability of this model, it is necessary to update the sample set by introducing new samples when they appear. Now, this approach and the corresponding dataset are in progress in our laboratory.

\section{Conclusions}

In this work, a quantitative model for moisture content measurement in walnut kernel was developed based on NIR diffuse reflectance spectroscopy using chemometric methods. The experimental results showed that suitable spectral pretreatment algorithms and band selection can significantly improve the prediction precision of the moisture content model. Through analysis, the optimized prediction model for walnut kernel moisture content can be established according to the following conditions: pretreatment method SNV + FD, band 1349-1490 nm, and PLS method. The values of $R^{2}$ and RMSEP of this model were 0.9865 and 0.0017 , showing that this model can accurately predict the moisture content in walnut kernel. The results also indicated that this method meets the requirements of routine food control and can be used to measure the moisture content of walnut kernel in practice. However, the moisture content of walnut kernel is susceptible to several factors such as variety, origin, cultivation management, and storage condition. Therefore, more efforts are needed to expand the size of the sample set to improve the performance and versatility of the model.

\section{Data Availability}

The data used to support the findings of this study are deposited in the Dryad repository (https://datadryad.org/ stash/share/Gv_u3jnm9QF0YB1xbZTC-krtuiHPX2ouqfmvUPMUe0).

\section{Conflicts of Interest}

The authors declare that they have no conflicts of interest.

\section{Authors' Contributions}

Dan Peng designed the study and interpreted the results. YaLi Liu and JiaSheng Yang collected the samples and got the associated spectra. Dan Peng, JingNan Chen, and Yanlan $\mathrm{Bi}$ made the programs using Matlab and tested the data. Yanlan Bi helped for drafting the manuscript. 


\section{Acknowledgments}

This work was supported by Key Scientific and Technological Project of Henan Province (212102110341) and the National Natural Science Foundation of China (31601537).

\section{References}

[1] D. O. Labuckas, D. M. Maestri, M. Perelló, M. L. Martínez, and A. L. Lamarque, "Phenolics from walnut (Juglans regia L.) kernels: antioxidant activity and interactions with proteins," Food Chemistry, vol. 107, no. 2, pp. 607-612, 2008.

[2] M. Kornsteiner, K.-H. Wagner, and I. Elmadfa, "Tocopherols and total phenolics in 10 different nut types," Food Chemistry, vol. 98, no. 2, pp. 381-387, 2006.

[3] F. Shahbazi, "Effects of moisture content and impact energy on the cracking characteristics of walnuts," International Journal of Food Engineering, vol. 10, pp. 165-181, 2014.

[4] H. Fathollahzadeh, H. Tabatabaie, and H. Mobli, "Effective conditions for extracting higher quality kernels from the sonnati salams apricot," International Journal of Food Engineering, vol. 6, pp. 61-64, 2010.

[5] Y. Zhao, L. Chen, W. Ji, J. Guo, and J. Wang, "Study on a novel energy-saving cryogenic pre-treatment equipment for walnut kernel peeling," Food Control, vol. 121, Article ID 107650, 2021.

[6] L. Wei, H. Fu, M. Lin et al., "Identification of dominant fungal contamination of walnut in Northwestern China and effects of storage conditions on walnut kernels," Scientia Horticulturae, vol. 264, Article ID 109141, 2020.

[7] H. Togrul and N. Arslan, "Moisture sorption isotherms and thermodynamic properties of walnut kernels," Journal of Stored Products Research, vol. 43, pp. 252-264, 2007.

[8] J. Wang, B. Gong, H. Ma, and J. H. Chen, "English words causing particular translation problems," Modern Welsh: A Comprehensive Grammar, vol. 48, pp. 490-496, 2015.

[9] C. K. Mutegi, H. K. Ngugi, S. L. Hendriks, and R. B. Jones, "Prevalence and factors associated with aflatoxin contamination of peanuts from Western Kenya," International Journal of Food Microbiology, vol. 130, no. 1, pp. 27-34, 2009.

[10] X. Wang, G. G. Atungulu, R. Khir, Z. Gao, and D. Slaughter, "Sorting in-shell walnuts by using near infrared spectroscopy for improved drying efficiency and product quality," International Agricultural Engineering Journal, vol. 26, pp. 165$172,2017$.

[11] I. M. Nolasco Perez, A. T. Badaró, S. Barbon, A. P. A. Barbon, M. A. R. Pollonio, and D. F. Barbin, "Classification of chicken parts using a portable near-infrared (NIR) spectrophotometer and machine learning," Applied Spectroscopy, vol. 72, no. 12, pp. 1774-1780, 2018.

[12] A. Fazayeli and S. Kamgar, S. M. Nassiri, H. Fazayeli, and M. de la Guardia, Dielectric spectroscopy as a potential technique for prediction of kiwifruit quality indices during storage," Information Processing in Agriculture, vol. 6, no. 4, pp. 479-486, 2019.

[13] M. Gabriele, D. Olesya, S. Tom, and S. Carsten, "Talanta," The International Journal of Pure and Applied Analytical Chemistry, vol. 188, pp. 91-98, 2018.

[14] S. B. Kim, C. Temiyasathit, K. Bensalah et al., "An effective classification procedure for diagnosis of prostate cancer in near infrared spectra," Expert Systems with Applications, vol. 37, no. 5, pp. 3863-3869, 2010.

[15] S. Barbon Junior, S. M. Mastelini, A. P. A. C. Barbon et al., "Multi-target prediction of wheat flour quality parameters with near infrared spectroscopy," Information Processing in Agriculture, vol. 7, no. 2, pp. 342-354, 2020.

[16] L.-G. Zhang, X. Zhang, L.-J. Ni, Z.-B. Xue, X. Gu, and S.-X. Huang, "Rapid identification of adulterated cow milk by non-linear pattern recognition methods based on near infrared spectroscopy," Food Chemistry, vol. 145, pp. 342-348, 2014.

[17] V. Wiedemair, R. Ramoner, and C. W. Huck, "Investigations into the total antioxidant capacities of cultivars of gluten-free grains using near-infrared spectroscopy," Food Control, vol. 95, pp. 189-195, 2019.

[18] G. Fox and M. Manley, "Applications of single kernel conventional and hyperspectral imaging near infrared spectroscopy in cereals," Journal of the Science of Food and Agriculture, vol. 94, no. 2, pp. 174-179, 2013.

[19] V. Sileoni, G. Perretti, L. Marte, O. Marconi, and P. Fantozzi, "Near-infrared spectroscopy for proficient quality evaluation of the malt and maize used for beer production," Journal of the Institute of Brewing, vol. 116, no. 2, pp. 134-139, 2012.

[20] D. S. Ferreira, O. F. Galão, J. A. L. Pallone, and R. J. Poppi, "Comparison and application of near-infrared (NIR) and mid-infrared (MIR) spectroscopy for determination of quality parameters in soybean samples," Food Control, vol. 35, no. 1, pp. 227-232, 2014.

[21] C. Miralbés, "Prediction chemical composition and alveograph parameters on wheat by near-infrared transmittance spectroscopy," Journal of Agricultural and Food Chemistry, vol. 51, no. 21, pp. 6335-6339, 2003.

[22] K. H. S. Peiris, Y. Dong, M. A. Davis, W. W. Bockus, and F. E. Dowell, "Estimation of the deoxynivalenol and moisture contents of bulk wheat grain samples by FT-NIR spectroscopy," Cereal Chemistry Journal, vol. 94, no. 4, pp. 677-682, 2017.

[23] G. Mishra, S. Srivastava, B. K. Panda, H. N. Mishra, and H. N. Mishra, "Rapid assessment of quality change and insect infestation in stored wheat grain using FT-NIR spectroscopy and chemometrics," Food Analytical Methods, vol. 11, no. 4, pp. 1189-1198, 2018.

[24] S. R. Delwiche, R. W. Higginbotham, and C. M. Steber, "Falling number of soft white wheat by near-infrared spectroscopy: a challenge revisited," Cereal Chemistry, vol. 95, no. 3, pp. 469-477, 2018.

[25] A. López, S. Arazuri, I. García, J. Mangado, and C. Jarén, “A review of the application of near-infrared spectroscopy for the analysis of potatoes," Journal of Agricultural and Food Chemistry, vol. 61, no. 23, pp. 5413-5424, 2013.

[26] R. Farhadi, A. H. Afkari-Sayyah, B. Jamshidi, and A. M. Gorji, "Prediction of internal compositions change in potato during storage using visible/near-infrared (Vis/NIR) spectroscopy," International Journal of Food Engineering, vol. 16, pp. 395424, 2020.

[27] B. Lu, X. Wang, N. Liu, K. He, and K. Wu, "Spectrochimica acta part A," Molecular and Biomolecular Spectroscopy, vol. 239, pp. 1386-1425, 2020.

[28] J. C. Hashimoto, J. C. Lima, R. M. S. Celeghini et al., "Quality control of commercial cocoa beans (theobroma cacao L.) by near-infrared spectroscopy," Food Analytical Methods, vol. 11, no. 5, pp. 1510-1517, 2018.

[29] M. K. Lakshmanan, T. Chinnu, and G. Arvamuthan, "Nearinfrared reflectance spectroscopy based online moisture measurement in copra," Journal of Food Process Engineering, vol. 43, no. 3, 2020. 
[30] J. Hu, X. Ma, L. Liu, Y. Wu, and J. Ouyang, "Rapid evaluation of the quality of chestnuts using near-infrared reflectance spectroscopy," Food Chemistry, vol. 231, pp. 141-147, 2017.

[31] Y. Zhang and W. Guo, "Moisture content detection of maize seed based on visible/near-infrared and near-infrared hyperspectral imaging technology," International Journal of Food Science \& Technology, vol. 55, no. 2, pp. 631-640, 2020.

[32] J. Zhang, L. Dai, and F. Cheng, "Classification of frozen corn seeds using hyperspectral VIS/NIR reflectance imaging," Molecules, vol. 24, no. 1, p. 149, 2019.

[33] B. Shi, L. Zhao, H. Wang, and D. Zhu, "Signal optimization approaches on the prediction of apples firmness by near infrared spectroscopy," Sensor Letters, vol. 9, no. 3, pp. 1062-1068, 2011.

[34] P. S. Sampaio, A. Soares, A. Castanho, A. S. Almeida, J. Oliveira, and C. Brites, "Optimization of rice amylose determination by NIR-spectroscopy using PLS chemometrics algorithms," Food Chemistry, vol. 242, pp. 196-204, 2018.

[35] J. Yi, Y. Sun, Z. Zhu, N. Liu, and J. Lu, "Near-infrared reflectance spectroscopy for the prediction of chemical composition in walnut kernel," International Journal of Food Properties, vol. 20, no. 7, pp. 1633-1642, 2016.

[36] Z. Xia, C. Zhang, H. Weng, P. Nie, and Y. He, "Sensitive wavelengths selection in identification of ophiopogon japonicus based on near-infrared hyperspectral imaging technology," International Journal of Analytical Chemistry, vol. 2017, pp. 1-11, Article ID 6018769, 2017.

[37] F. Allegrini and A. C. Olivieri, "Multi-way figures of merit in the presence of heteroscedastic and correlated instrumental noise: unfolded partial least-squares with residual multi-linearization," Chemometrics and Intelligent Laboratory Systems, vol. 158, pp. 200-209, 2016.

[38] L. Salguero-Chaparro, V. Baeten, J. A. Fernández-Pierna, F. Peña-Rodríguez, and P. R. Francisco, "Near infrared spectroscopy (NIRS) for on-line determination of quality parameters in intact olives," Food Chemistry, vol. 139, no. 1-4, pp. 1121-1126, 2013. 